\title{
Development of Navigation Control Algorithm for AGV Using D* search Algorithm
}

\author{
Jeong Geun Kim ${ }^{\# 1}$, Dae Hwan Kim ${ }^{\$ 2}$, Sang Kwun Jeong ${ }^{* 3}$, Hak Kyeong Kim ${ }^{\# 4}$ Sang Bong Kim ${ }^{\# 5}$ \\ ${ }^{\#}$ Department of Mechanical and Automotive Eng., Pukyong National University, Busan 608-739, Korea \\ ${ }^{5} \mathrm{kimsb} @ \mathrm{pknu} \cdot \mathrm{ac} \cdot \mathrm{kr}$ \\ ${ }^{\$}$ Department of Fluid Mechanics Engineering, Friedrich Alexander University, Busan campus 1276, Korea \\ *Han Sung Well Tech Co, Ltd, Busan 720-20, Korea
}

\begin{abstract}
In this paper, we present a navigation control algorithm for Automatic Guided Vehicles (AGV) that move in industrial environments including static and moving obstacles using $D^{*}$ algorithm. This algorithm has ability to get paths planning in unknown, partially known and changing environments efficiently. To apply the $D^{*}$ search algorithm, the grid map represent the known environment is generated. By using the laser scanner LMS-151 and laser navigation sensor $N A V-200$, the grid map is updated according to the changing of environment and obstacles. When the AGV finds some new map information such as new unknown obstacles, it adds the information to its map and re-plans a new shortest path from its current coordinates to the given goal coordinates. It repeats the process until it reaches the goal coordinates. This algorithm is verified through simulation and experiment. The simulation and experimental results show that the algorithm can be used to move the $A G V$ successfully to reach the goal position while it avoids unknown moving and static obstacles.
\end{abstract}

[Keywords - navigation control algorithm; Automatic Guided Vehicles (AGV); $D^{*}$ search algorithm]

\section{INTRODUCTION}

As factory automation has been progressed, logistics systems have required increasing intelligence and flexibility. Automated guided vehicle (AGV) currently uses in logistics systems features high flexibility and easy configurability. Nowadays the most popular navigation method for $\mathrm{AGV}$ is trajectory tracking navigation method such as in [17]. This navigation method follows the given path, but unresponsive to unknown obstacle on AGV's trajectory. P.S. Pratama et al [18] was developed the obstacle avoidance algorithm for unknown environment. However, the path is not optimal because the optimal trajectory is not planned before the AGV moved.

To navigate the AGV from one point to another point optimally, the path planning algorithm is needed. To generate the optimal path from start to goal position using given map, configuration space method [1], potential field [2-5] and gradient method (GM) [6] were proposed. Those algorithms are called global path planning. Those algorithms work only for known and static environments. Furthermore, if there are many obstacles, complex structures and suddenly path changing, those algorithms spend a lot of energy and calculation time.

To deal with dynamic environment, VFH (Vector Field Histogram) [7], CVM (Curvature Velocity Method) [8], Fuzzy rule [9], MPC (Model Predictive Control) [10], DWA (Dynamic Window Approach) [11-13] have been researched. Those algorithms generate the optimal path in local area based on the environment information obtained from the sensor. Those algorithms are called local path planning. Those algorithms find the obstacles using real-time processing.
Those algorithms deal with unknown obstacle therefore suitable for obstacles avoidance. On the other hand, because the calculated area is limited, the goal position is unreachable. Therefore, an algorithm that combines both path planning algorithms is needed.

This paper proposes a fusion algorithm using the $\mathrm{D}^{*}$ search algorithm that combines global path planning and the local path planning algorithm for collision avoidance algorithm. D* search algorithm is possible to make global path planning and the local path planning at same time, so the fixed obstacles or unexpected obstacles are successfully detected and avoided and destination point can be reached quickly. To verify the effectiveness of proposed algorithm, simulation and experiment are done. The simulation and experimental result shows that the proposed algorithm successfully generates the optimal path using global path planning and local path planning.

\section{PATH PLANNING}

\section{A. Algorithm Description}

The D* algorithm was first introduced by Stentz [14]. The name of the algorithm, $\mathrm{D}^{*}$, was chosen because it resembles $A^{*}$ [15], except that it is dynamic in the sense that arc cost parameters can change during the problem solving process. $\mathrm{A}^{*}$ algorithm is widely used in off-line path planning and robot motion planning. The $\mathrm{D}^{*}$ is path length optimal algorithm and saves a lot of computational time compared with $\mathrm{A}^{*}$. Provided that robot motion is properly coupled to the algorithm, D* generates optimal trajectories.

Local path re-planning process is needed at the global planning in the situation of partially known environment. According to the difference of original point between the replanning path and the original planning path, there are two kinds of re-planning, including completion re-planning and the incompletion re-planning. Completion re-planning has the same original point between re-planning path and original planning path. Incompletion re-planning has different original point between re-planning path and original planning path. D* algorithm is a kind of incompletion re-planning. But it uses the original planning information. So it combines the characters of the optimization and the real-time path planning. $\mathrm{D}^{*}$ is able to use the global planning and local information. And it is also good at utilizing the off-line planning and online planning. 


\section{B. Global Path Planning}

Global path planning method in $\mathrm{D}^{*}$ is similar with $\mathrm{A}^{*}$ algorithm except the direction of calculation. A shortest path in $A^{*}$ can be computed forwards, from origin to destination. On the other hand, in $\mathrm{D}^{*}$, it is computed backwards, from destination to origin. The robot starts at a particular state SP and moves across arcs (incurring the cost of traversal) to other states until it reaches the goal state, denoted by $G$. Every state except $G$ has a back pointer to a next state $Y$ denoted by $b(X)=Y$. D* uses back pointers to represent paths to the goal. The cost of traversing an arc from state $Y$ to state $X$ is a positive number given by the arc cost function $c(X, Y)$. If $Y$ does not have an arc to $X, c(X, Y)$ is undefined. Two states $X$ and $Y$ are neighbours in the space if $c(X, Y)$ or $c(X, Y)$ is defined. Like A*, $\mathrm{D}^{*}$ maintains an OPEN list of states. The OPEN list is used to propagate information about changes to the arc cost function and to calculate path costs to states in the space. Every state $Y$ has an associated tag $t(Y)$, such that $t(Y)=N E W$ if $Y$ has never been on the OPEN list, $t(Y)=O P E N$ if $Y$ is currently on the OPEN list, and $t(X)=C L O S E D$ if $Y$ is no longer on the $O P E N$ list. For each state $X, \mathrm{D}^{*}$ maintains an estimate of the sum of the arc costs from $X$ to $G$ given by the path cost function $h(G, X)$. For each state $X$ on the OPEN list (i.e., $t(Y)=O P E N)$, the key function, $k(G, X)$, is defined to be equal to the minimum of $h(G, X)$ before modification and all values assumed by $h(G, X)$ since $X$ on the OPEN list. The parameter $k_{\text {min }}$ is defined to be $\quad(\mathrm{k}(\mathrm{X}))$ for all $X$ such that $t(X)=O P E N$. The parameter $k_{\text {min }}$ represents an important threshold in $\mathrm{D}^{*}$ : path costs less than or equal to $k_{\min }$ are optimal, and those greater than $k_{\min }$ may not be optimal. The parameter $\mathrm{k}$ is defined to be equal to $k_{\min }$ prior to most recent removal of a state from the OPEN list.

The optimal path for global path planning can be obtained using D* algorithm flow chart as shown in Fig.1.

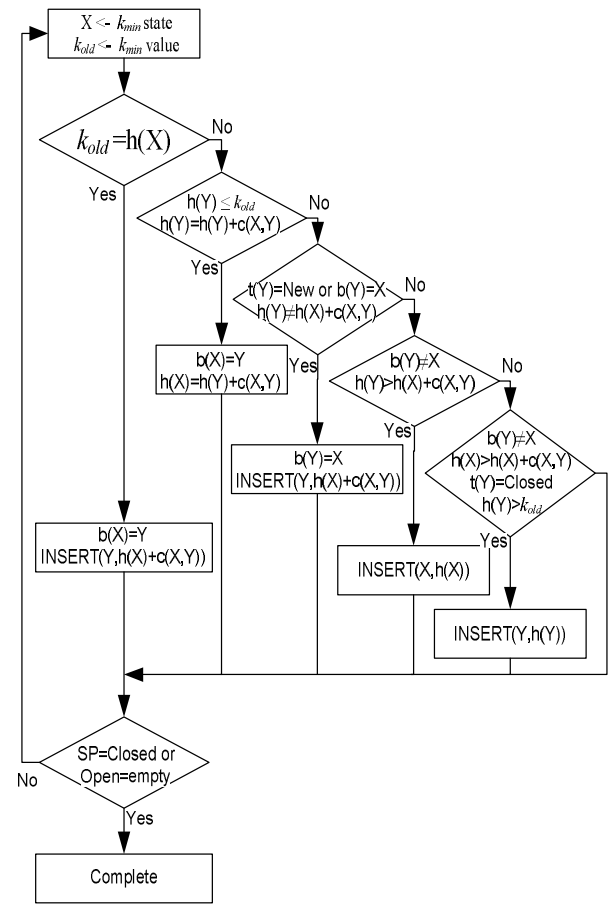

Fig. 1 Complete flowchart of $\mathrm{D}^{*}$ algorithm

\section{Local Path Planning}

Fig. 2 shows the condition when $\mathrm{D}^{*}$ re-planning the path. When an obstacle is detected, $\mathrm{A}^{*}$ and $\mathrm{D}^{*}$ algorithms restart the general search process. This is equivalent to find a "broken" $\operatorname{arc} A$ in an initial path $i$ of a graph. $i$ is a time or length optimal path between an starting node and a goal node

- Arc $A$ is defined by two nodes: (current node), which represents the current robot position and $n$ (next node), which represents the next robot's movement destination. Nodes $\{, \quad$, and $n\} \in N$. Every node connected to the current node , except $n$, is used to generate a new partial solution path set. Each new path new from the new path set is added to the $\mathrm{D}^{*} O P E N$ list. The $O P E N$ list contains a set of partial solution paths that are expanded until is reached. Thus, a first path/node expansion implies to generate new partial solution paths new composed of nodes included in ${ }_{i}^{*}=(,, \quad) \subset i$ and the set of neighbour nodes connected to . Arc $A$ is ignored and cannot be a part of any solution.
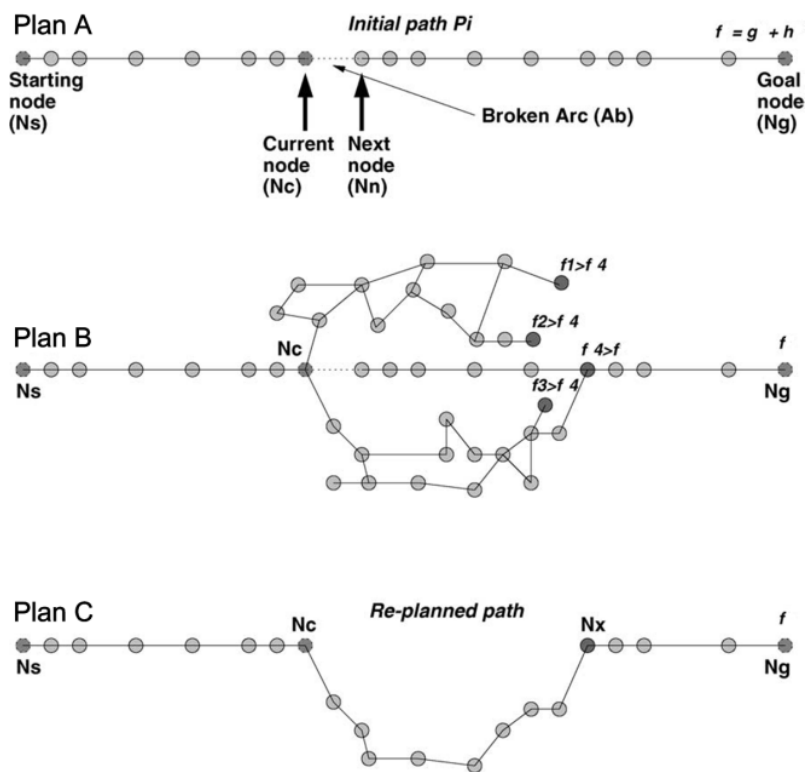

Fig. 2 D* re-planning path

Plan A shows an initial path $i . \quad$ is the current node and $A$ the "broken" arc. Plan B shows the algorithm to find the path until it reaches the goal. Plan $\mathrm{C}$ shows final re-planed path using $\mathrm{D}^{*}$ algorithm.

The whole search process ends when:

1) $n$ or another node included in the original path $i$ is found and the rest of partial paths (solutions) included in the $O P E N$ list have a higher f value. $f=g+h$, where $g$ is the accumulated path cost and $h$ is the heuristic value that estimates path cost from to (see Fig. 2). Usually, function $f$ implements the Euclidean distance.

2) The goal node is reached and the rest of partial paths have a higher $f$ value. This is equivalent to a brute-force method and represents the worst case.

3) The OPEN list is empty. This means that there are no solutions without $A$.

An example of local path planning is shown in Fig. 3 
Columns

Rows \begin{tabular}{l|l|l|l|l|l|l|l|l|l|} 
& 1 & 2 & 3 & 4 & 5 & 6 & 7 & 8 & 9 \\
\hline 1 & $x$ & & & & & & & & \\
\hline 2 & & & & & & & & & \\
\hline 3 & & & & & & & & & \\
\hline 4 & & & $x$ & & & & & & \\
\hline 5 & & & & & & & & & \\
\hline 6 & & & & & & & & & \\
\hline 7 & & & & & & $x$ & & & \\
\hline 8 & & & & & & & & & \\
\hline 9 & & & & & & & & & $x$ \\
\hline
\end{tabular}

Fig. 3 Example of local path planning

Fig. 3 shows path-planning example for local obstacle avoidance applied on a subsection of the search space.

\section{SYSTEM CONFIGURATION}

Fig. 4 shows the system configuration for experiment in this paper. Tank-800 industrial computer is used for main controller. Half PCI MMC Board connects the industrial computer with motor driver, all inputs and outputs. 200W/3000 rpm BLDC motor are used to move the driving wheel. For monitoring, LCD screen is used. All of those systems are powered by $2 \mathrm{x} 12 \mathrm{~V} / 8 \mathrm{Ah}$ batteries.

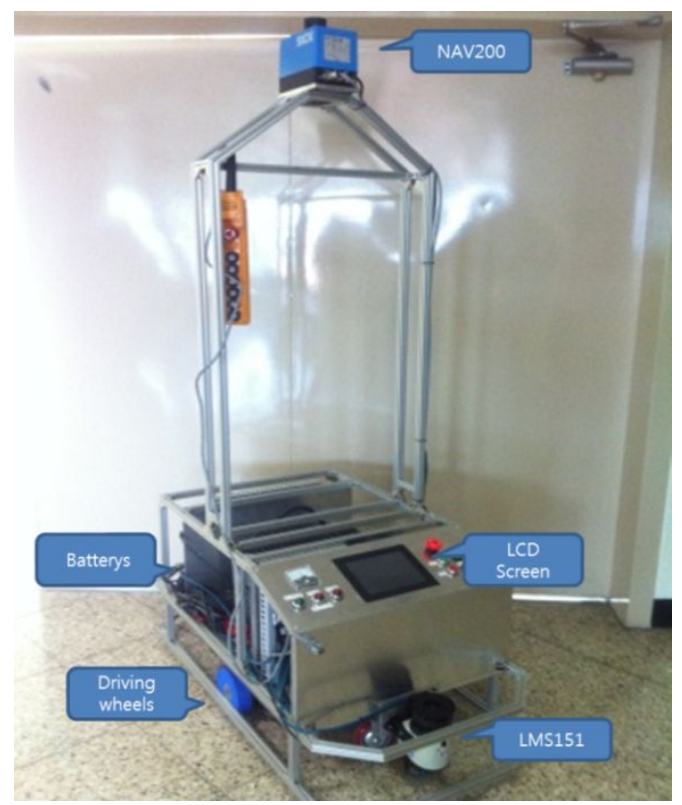

Fig. 4 AGV system

For positioning the AGV in absolute coordinate, NAV200 is used. NAV200 system is a laser-based positioning system that returns an absolute position of the scanner with respect to a user-defined local coordinate frame. Nav200 system comprises of two main units, a laser scanner unit and a Transputer Position Unit (TPU). This laser scanner continuously rotates 360 degrees to scan the reflectors and then sends the information to the TPU to compute the scanner's position. This system can provide accuracy up to 1 $\mathrm{mm}$ with an update rate up to $8 \mathrm{~Hz}$ [16]. The NAV200 navigation system is shown in Fig. 5.

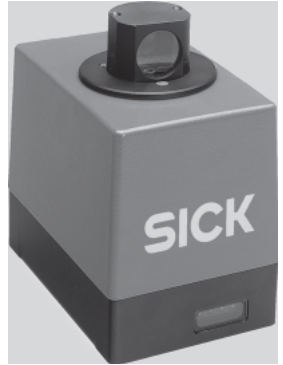

Fig.5 NAV200 laser navigation scanner

For obstacle detection, the laser measurement system LMS151 is employed. LMS151 system is a laser measurement system that recognizes obstacles in 270 degrees scan. The LMS151 is an electro-sensitive distance measurement system for stand-alone or network operation. It is suitable for applications in which precise, electro-sensitive measurements of contours and surroundings are required. It is also possible to realize systems, for instance, for collision protection, for building surveillance or for access monitoring. Scanning range up to $50 \mathrm{~m}$ and rotation frequency is $25 \mathrm{~Hz}$. The LMS151 measurement system is shown in Fig. 6.

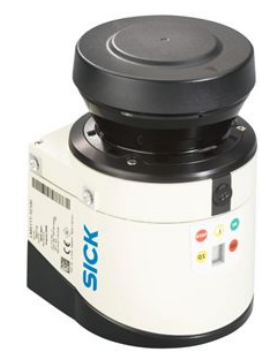

Fig. 6 LMS151 laser measurement scanner

\section{SIMULATION AND EXPERIMENTAL RESULTS}

\section{A. Simulation Results}

Simulation is done to confirm the feasibility of the D* Algorithm as shown in Fig.7.

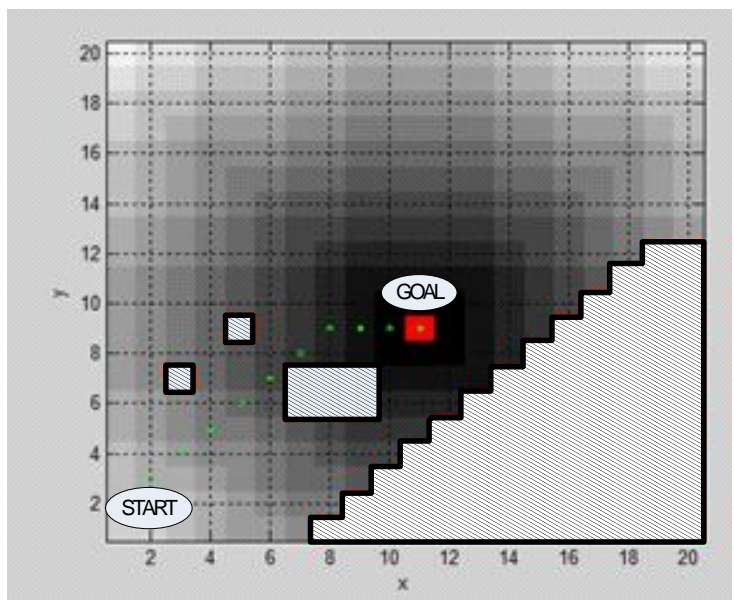

Fig.7 Simulation result for path planning

The map is obtained by converting the real environment into simple grid map. In this simulation, each grid is represented as one unit square. To simplify the calculation, in 
this simulation, one grid size $30 \mathrm{~cm} \times 30 \mathrm{~cm}$ is chosen. The optimal path that is calculated by $\mathrm{D}^{*}$ algorithm is shown in Fig.7.The slashed area represents the known obstacle in environment. The darker area represents the higher cost, and the lighter area represents the lower cost. The dot line represents the shortest trajectory from start position to the goal position.

\section{B. Experimental Results}

Fig. 8 shows the comparison between simulation result and real experiment result. Fig. 8 shows that the simulation result is similar with experimental result. They show that the proposed algorithm successfully generates the optimal path and the $\mathrm{AGV}$ can reach the goal position without colliding with the obstacles. Numbers on $\mathrm{x}$ axis and $\mathrm{y}$ axis in Fig. 8 is in grid number

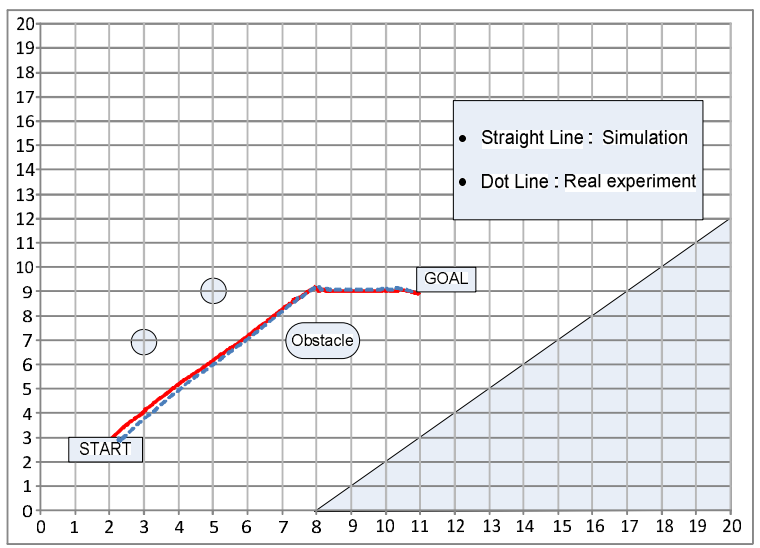

Fig. 8 Simulation and experimental results using the proposed algorithm

Fig. 9 shows the error between simulation and real experimental result.

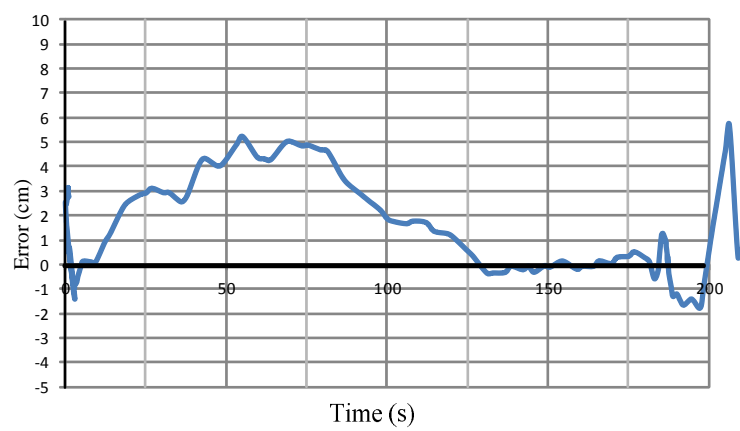

Fig. 9 Error between simulation and experimental results

The error between simulation and experimental results is defined as:

$e^{-} \quad e^{-}$

Where, e is position error, , are AGVposition from simulation result and $e, e$ are $\mathrm{AGV}$ position from experimental result. The error is bounded within $\pm 5 \mathrm{~cm}$. Therefore, it shows that the proposed algorithm can be successfully implemented in real system.

Fig. 10 shows the experimental results using $D^{*}$ algorithm. Fig. 10(a) shows the experimental AGV using the laser scanner LMS-151 and laser navigation sensor NAV-200 in start position. The AGV calculates the optimal path to the goal position using $\mathrm{D}^{*}$ algorithm. After generating the optimal path, the AGV moves forward while it is scanning the environment to update the information according to changing of environment and obstacle as shown in Fig. 10(b). When AGV finds some new obstacles that are different from the given map information such as unknown obstacle as shown in Fig. 10(c), it adds the information to its map and re-plans a new shortest path from its current coordinates to the given goal coordinates as shown in Figs. 10(d) (e). It repeats the process until it reaches the goal position as shown in Fig. 10(f).

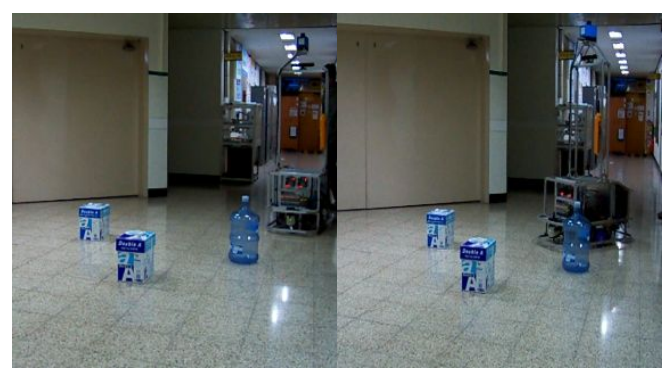

(a)

(b)

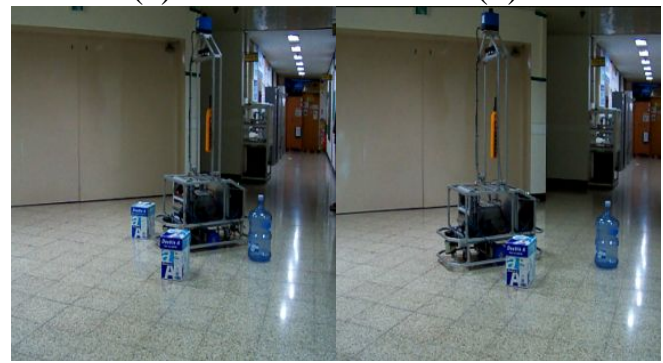

(c)

(d)

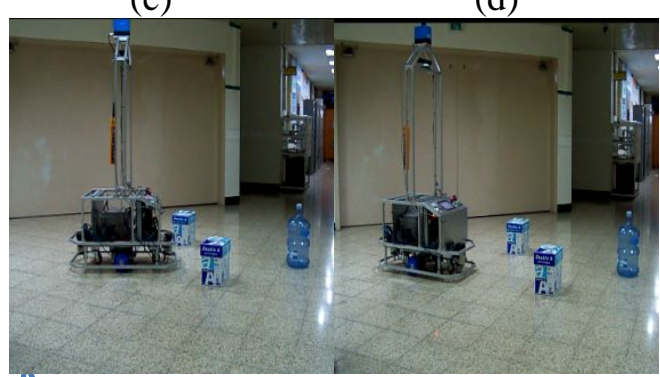

(e)

(f)

Fig. 10 Experimental Results

V. CONCLUSIONS

In this paper, global path planning and local path planning of AGV using $\mathrm{D}^{*}$ algorithm were presented. Using lasers positioning and laser measurement system, the position of $\mathrm{AGV}$ and the information of environment were updated to avoid the collision with obstacles. In $\mathrm{D}^{*}$ search algorithm when unknown obstacles were detected or the environment is changed, only the affected area was calculated to find the new path. Therefore, the path is fixed rapidly. The simulation and experimental results show that the algorithm successfully generates the optimal path and the AGV can reach the goal position without colliding with the obstacles. Furthermore, because the error between simulation and experimental result is small within $\pm 5 \mathrm{~cm}$, the proposed algorithm is effective and applicable to real workspace. 


\section{ACKNOWLEDGMENT}

This research was supported by a grant from Construction Technology Innovation Program (CTIP) funded by Ministry of Land, Transportation and Maritime Affairs (MLTM) of Korean government.

\section{REFERENCES}

[1] T. Loazno-Perez and M.A. Weslsy, "An Algorithm for Planning Collision-free Paths among Polyhedrial Obstacles," Commun. ACM, pp. 560-570,1979.

[2] O. Khatib, "Real-Time Obstacle Avoidance for manipulator and Mobile Robots," The International Journal of Robotic Research, MIT Press, Cambridge, pp. 90-98,1986.

[3] E. Rimon and E.E. Koditschek, "Exact Robot Navigation Using Artificial Potential Functions," IEEE Transactions on Robotics and Automation, Vol. 8, No. 5, pp. 501-518,October, 1992.

[4] H.I. Yoo and B.S. Kang, "Precise Motion Control of a Mobile Robot Based on Potential Field Method," The KSME 2008 Dynamics and Control spring annual meeting, pp. 177-180, 2008.

[5] M.G. Park and M.C. Lee, "A New Technique to Escape Local Minimum in Artificial Potential Field Based Path Planning," KSME International Journal, Vol.17, No.12,pp. 1876-1885, 2003.

[6] J. Bruce and M. Veloso, "Real-Time Randomized Path Planning for Robot Navigation," Proc. of the 2002 IEEE/RSJ Int. Conference on Intelligent Robots and Systems EPFL, Lausanne, Switzerland,pp. 2383-2388,October, 2002.

[7] K. Konolige, "A Gradient Method for Realtime Robot Control," Proc of International Conf. on Intelligent Robots and Systems, pp. 639-646, 2000 .

[8] J. Borenstein and Y. Koren, "The Vector Field Histogram-Fast Obstacle Avoidance for mobile Robots," IEEE Journal of Robotics and Automation Vol. 7, No. 3, pp. 278-288, June 1991.
[9] D. Fox, W. Burgard and S. Thrun, "The Dynamic Window Approach to Collision Avoidance," IEEE Robotics and Automation, Vol. 4, No. 1,pp. 23-33, 1997.

[10] R. Simmons, "The Curvature-Velocity Method for Local Obstacle Avoidance," Proc. Of International Conf. on Robotics and Automation, pp. 3375-3382,1996.

[11] H.J. Kim, D.H. Shim and S. Sastry, "Nonlinear Model Predictive Tracking Control for Rotorcraft-Based Unmanned Aerial Vehicles," Proceedings of the American Control Conference, Anchorage, AK pp 8-10,May, 2002.

[12] P. Ogren and N.E. Leonard, "A Convergent Dynamic Window Approach to Obstacle Avoidance," IEEE Transactions on Robotics and Automation, pp. 188-195, 2003.

[13] C. Stachniss and W. Burgard, "An Integrated Approach to GoalDirected Obstacle Avoidance Under Dynamic Constraints for Dynamic Environments," Proceedings of the 2002 IEEE/RSJ, intl, Conference on Intelligent Robots and Systems EPFL, Lausanne, Switzerland, pp. 508-513, October, 2002.

[14] A. Stentz, "Optimal and Efficient Path Planning for Partially-known Environments", in: Proceedings of the IEEE International Conference on Robotics and Automation (ICRA'94), Vol. 4, pp. 3310-3317, 1994.

[15] N.J. Nilsson, "Principles of Artificial Intelligence", Tioga Publishing Company, pp. 76-79, 1980.

[16] "NAV200 Positioning System for Navigation Support Technical Description," SICK

[17] T.L. Bui et al, "AGV Trajectory Control Based on Laser Sensor Navigation", International Journal of Science and Engineering, Vol. 4(1), pp. 39-43, 2013.

[18] P.S. Pratama, Sang K..J., Soon S.P, S.B. Kim, "Moving Object Tracking and Avoidance Algorithm for Differential Driving AGV Based on Laser Measurement Technology", International Journal of Science and Engineering, Vol. 4(1), pp. 11-15, 2013. 